\title{
BMJ Open Pilot investigation of the oxygen demands and metabolic cost of incremental shuttle walking and treadmill walking in patients with cardiovascular disease
}

\author{
M Almodhy, ${ }^{1}$ R Beneke, ${ }^{2}$ F Cardoso, ${ }^{1}$ M J D Taylor, ${ }^{1}$ G R H Sandercock ${ }^{1}$
}

To cite: Almodhy M, Beneke R, Cardoso F, et al. Pilot investigation of the oxygen demands and metabolic cost of incremental shuttle walking and treadmill walking in patients with cardiovascular disease. BMJ Open 2014;4:e005216. doi:10.1136/bmjopen-2014005216

- Prepublication history for this paper is available online. To view these files please visit the journal online (http://dx.doi.org/10.1136/ bmjopen-2014-005216).

Received 10 March 2014 Revised 9 August 2014 Accepted 11 August 2014

CrossMark

${ }^{1}$ Centre for Sports \& Exercise Sciences, School of Biological Sciences, University of Essex, Colchester, UK

${ }^{2}$ Institut für

Sportwissenschaft und Motologie Medezin, PhilippsUniversität Marburg,

Marburg, Germany

Correspondence to Dr G R H Sandercock; gavins@essex.ac.uk

\section{ABSTRACT}

Objective: To determine if the metabolic cost of the incremental shuttle-walking test protocol is the same as treadmill walking or predicted values of walkingspeed equations.

Setting: Primary care (community-based cardiac rehabilitation).

Participants: Eight Caucasian cardiac rehabilitation patients (7 males) with a mean age of $67 \pm 5.2$ years. Primary and secondary outcome measures: Oxygen consumption, metabolic power and energy cost of walking during treadmill and shuttle walking performed in a balanced order with 1 week between trials.

Results: Average overall energy cost per metre was higher during treadmill walking $(3.22 \pm 0.55 \mathrm{~J} \mathrm{~kg} / \mathrm{m})$ than during shuttle walking $(3.00 \pm 0.41 \mathrm{~J} \mathrm{~kg} / \mathrm{m})$. There were significant post hoc effects at $0.67 \mathrm{~m} / \mathrm{s}(p<0.004)$ and $0.84 \mathrm{~m} / \mathrm{s}(\mathrm{p}<0.001)$, where the energy cost of treadmill walking was significantly higher than that of shuttle walking. This pattern was reversed at walking speeds $1.52 \mathrm{~m} / \mathrm{s}(\mathrm{p}<0.042)$ and $1.69 \mathrm{~m} / \mathrm{s}(\mathrm{p}<0.007)$ where shuttle walking had a greater energy cost per metre than treadmill walking. At all walking speeds, the energy cost of shuttle walking was higher than that predicted using the American College of Sports Medicine walking equations.

Conclusions: The energetic demands of shuttle walking were fundamentally different from those of treadmill walking and should not be directly compared. We warn against estimating the metabolic cost of the incremental shuttle-walking test using the current walking-speed equations.

\section{INTRODUCTION}

Since its conception as an alternative to incremental treadmill testing of patients with chronic obstructive pulmonary disease, the incremental shuttle-walking test (ISWT) has gained popularity as an estimate of functional capacity in numerous clinical populations. The ISWT appears adequately reliable ${ }^{1}$

\section{Strengths and limitations of this study}

- We provide the first direct comparison of the metabolic cost of shuttle walking and treadmill walking in cardiac patients.

- Our data suggest that metabolic demands of these exercise modalities appear fundamentally different.

- We suggest that current methods to estimate the metabolic cost of shuttle walking are flawed. We warn against risk stratification of cardiac patients based on estimated oxygen costs using the American College of Sports Medicine walking equations during the incremental shuttle-walking test.

- The sample size limits generalisability, particularly in female patients who are not represented at higher walking speeds-a larger study of metabolic cost of the incremental shuttle-walking test is warranted.

and is sensitive to changes in functional capacity. $^{2}{ }^{3}$ However, the ISWT's validity as an estimate of cardiovascular fitness is only moderate, ${ }^{3}$ and the use of the test to estimate oxygen consumption exercise capacity in metabolic equivalents (METs) is questionable. $^{4}$

Woolf-May and Ferret ${ }^{4}$ reported acceptable agreement between the energy cost of treadmill walking and the ISWT in healthy volunteers using linear regression analyses, but did not assess this relationship in cardiac patients. The authors reported higher energy demands of shuttle walking in cardiac patients compared with healthy controls. They suggested this may be due to poorer walking economy in the former; they did not report walking economy during ISWT or make comparisons between shuttle-walking and treadmill-walking economy. 
Treadmill-walking and shuttle-walking tests are routinely used to assess patients with cardiovascular disease and we have previously reported discrete values for change in fitness measured using these tests. ${ }^{5}$ Prior to undertaking a proposed multicentre study to identify predictors of change in cardiorespiratory fitness due to cardiac rehabilitation, we performed the present pilot study. We examined whether there were differences in the metabolic demands and energy cost of treadmill and shuttle walking in cardiac rehabilitation patients in order to determine whether we could combine data from these tests in our multicentre study. We also compared metabolic cost of the ISWT with values predicted from treadmill-walking equations ${ }^{6}$ and published estimates. ${ }^{4}$

\section{METHODS}

Participants ( $\mathrm{n}=8 ; 7$ males; $67 \pm 5.2$ years: $86.6 \pm 10.1 \mathrm{~kg}$ ) were stable cardiac patients attending community-based rehabilitation following elective cardiac revascularisation. All patients gave written, informed consent.

\section{Equipment}

The ISWT was performed on a non-slip floor using two cones placed $9 \mathrm{~m}$ apart and a portable CD player. The treadmill test was performed on a motorised treadmill (Quaser, HP Cosmos, Nussdorf, Germany). During both tests a portable gas analyser (K4b2 Mobile Breath by Breath Metabolic System, COSMED Pulmonary Function Equipment, Rome, Italy) was used to record expired gas collected via a face and nose mask (Hans Rudolph, Shawnee, Kansas, US A). This was calibrated using gases of a known concentration and a syringe before each test.

\section{Protocol}

Patients completed the ISWT and the treadmill test in a balanced order with 1 week between trials. The ISWT was performed in accordance with national recommendations for cardiac patients. ${ }^{8}$ Briefly, the 12-stage protocol starts at a walking speed of $0.5 \mathrm{~m} / \mathrm{s}(1.12 \mathrm{mph})$ and increases by $0.17 \mathrm{~m} / \mathrm{s}(0.38 \mathrm{mph})$ each minute. An identical incremental protocol was programmed into the treadmill. Patients were accustomed to treadmill walking but received a brief period of familiarisation in which they were required to walk without holding the treadmill handles before the ISWT protocol was also performed.

\section{Calculation of metabolic power and energy cost of walking}

We assumed a standard resting metabolic rate of $4 \mathrm{~mL} /$ $\mathrm{kg} / \mathrm{min}$ based on reference standards. ${ }^{9}$ Metabolic power was then calculated via indirect calorimetry from $\dot{\mathrm{V}}_{2}$ and $\dot{\mathrm{V}} \mathrm{CO}_{2}$ above rest and from body mass: metabolic power $[\mathrm{W} / \mathrm{kg}]=\left(\dot{\mathrm{V}} \mathrm{O}_{2}-\dot{\mathrm{V}} \mathrm{O}_{2 \text { rest }}\right)[\mathrm{mL} / \mathrm{kg} / \mathrm{s}]$ respiratory exchange ratio adjusted caloric equivalent $[\mathrm{J} / \mathrm{mL}] .{ }^{10}{ }^{11}$ To analyse the relationship between speed and metabolic power of walking, the metabolic power was predicted as a quadratic function of speed: metabolic power $=\mathrm{a}+\mathrm{b} \mathrm{v}^{2} .^{10}{ }^{12-16}$ The energy cost of walking per metre distance was calculated by: energy cost $[\mathrm{J} / \mathrm{kg} / \mathrm{m}]$ $=$ metabolic power $[\mathrm{W} / \mathrm{kg}] /$ speed $[\mathrm{m} / \mathrm{s}] .{ }^{10} 1117$

\section{Statistical analyses}

Descriptive results are presented as mean $\pm \mathrm{SD}$. A test modality-by-walking speed analysis of variance (ANOVA) with shuttle versus treadmill walking as within-participants factor and walking speed as the between-participants factor was performed. Significant interactions and main effects were further analysed using one-way ANOVA and paired samples t tests as appropriate. Based on the classical descriptions of walking energy cost, ${ }^{18-20}$ non-linear regression models were chosen to identify significant inter-relationships between metabolic power, energy cost per metre and walking speed, respectively. All analyses were completed using SPSS V.19.0 (SPSS Inc and IBM Company, Chicago, Illinois, USA) and statistical significance was defined as $\mathrm{p}<0.05$.

\section{RESULTS}

Figure 1 shows the oxygen uptake at each of seven stages completed by at least seven patients. There was a significant main effect for walking speed on oxygen uptake and a significant interaction between treadmill walking and shuttle walking on the ground. Oxygen uptake was higher in treadmill walking than shuttle walking at $0.67 \mathrm{~m} / \mathrm{s}(\mathrm{p}=0.006 ; \mathrm{n}=8)$ and $0.84 \mathrm{~m} / \mathrm{s}(\mathrm{p}=0.003 ; \mathrm{n}=8)$ but the significantly steeper increases in oxygen demand during shuttle walking meant the opposite was true at $1.69 \mathrm{~m} / \mathrm{s}(\mathrm{p}<0.006 ; \mathrm{n}=7)$.

Figure 2 shows the metabolic power of treadmill walking and shuttle walking. There was a main effect for walking speed on metabolic power during treadmill as well as shuttle walking $(\mathrm{p}<0.05)$. The different effects of walking modality on metabolic power were more pronounced if power was predicted as a function of walking

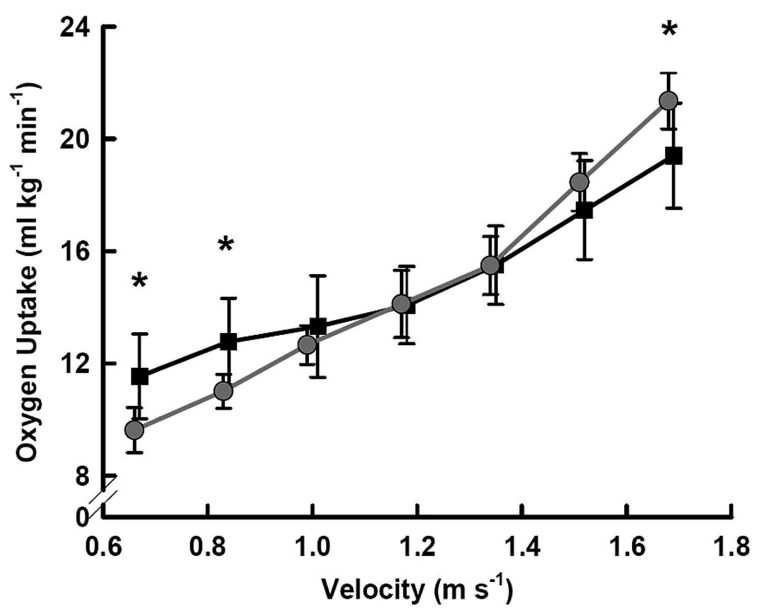

Figure 1 The oxygen uptake of treadmill walking (black line) and shuttle walking (grey line) at each of the seven stages; * treadmill walking different from shuttle walking, $p<0.05$. 


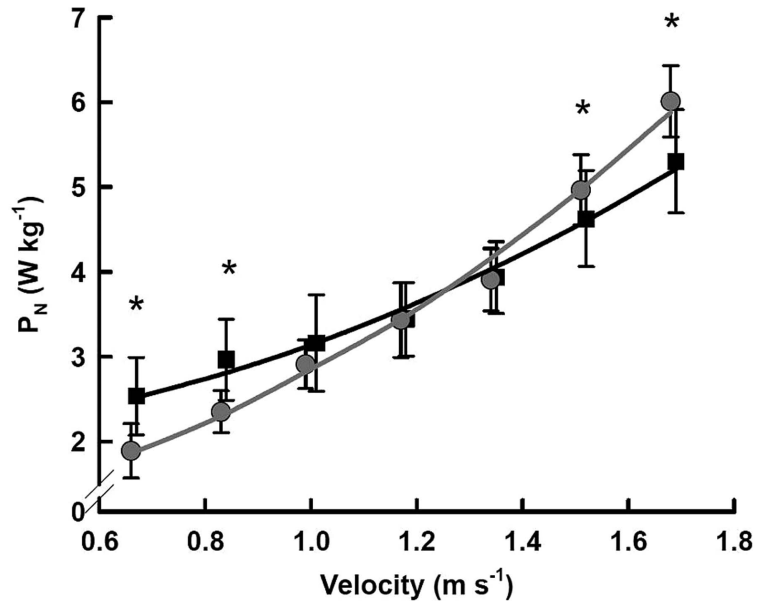

Figure 2 Metabolic power above rest $\left(\mathrm{P}_{\mathrm{N}}\right)$ of treadmill walking (black line) and shuttle walking (grey line) at each of the seven stages, * treadmill walking different from shuttle walking, $p<0.05$.

speed with power treadmill walking $=2.028+1.115 \mathrm{v}^{2}$ and power shuttle walking on the ground=1.126+1.665 $\mathrm{v}^{2}$ where $99 \%$ of the variance of power was explained by the quadratic curve fits in both modalities (both $\mathrm{p}<0.001)$. The difference in response to each modality was indicated by a significant interaction between modality and speed. There were significantly higher metabolic power requirements for treadmill walking at $0.67 \mathrm{~m} / \mathrm{s}$ $(\mathrm{p}<0.004 ; \mathrm{n}=8)$ and $0.84 \mathrm{~m} / \mathrm{s}(\mathrm{p}<0.001 ; \mathrm{n}=8)$. Owing to the steeper increase observed in shuttle walking the metabolic power was significantly higher at $1.52 \mathrm{~m} / \mathrm{s}$ $(\mathrm{p}=0.042 ; \mathrm{n}=7)$ and $1.69 \mathrm{~m} / \mathrm{s}(\mathrm{p}=0.007 ; \mathrm{n}=7)$ compared with treadmill walking.

Figure 3 shows the relative energy cost (per metre) of walking for both modalities. There were significant main effects for modality and speed in relative energy cost of

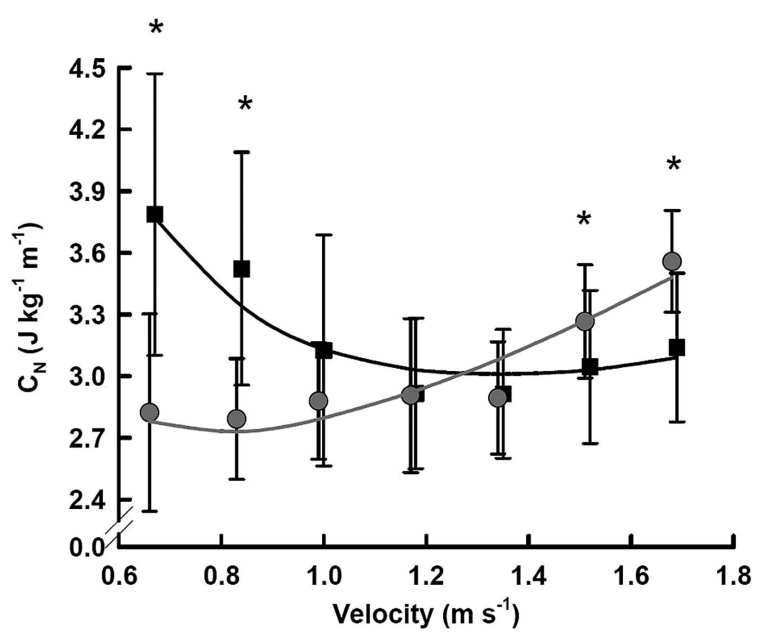

Figure 3 Energy cost above rest $\left(\mathrm{C}_{\mathrm{N}}\right)$ per metre distance of treadmill walking (black line) and shuttle walking (grey line) at each of the seven stages, * treadmill walking different from shuttle walking, $p<0.05$. walking, which was well described as a function of speed by the above approximated parameters for both walking modalities (energy cost treadmill walking $=2.028 / \mathrm{v}+1.115$ $\mathrm{v}$ and energy cost shuttle walking on the ground $=1.126$ / $\mathrm{v}+1.665 \mathrm{v}$; both $\mathrm{p}<0.001)$. Average overall energy cost per metre $(\mathrm{kg} / \mathrm{m})$ was higher during treadmill walking $(3.22$ $\pm 0.55 \mathrm{~J} \mathrm{~kg} / \mathrm{m})$ than during shuttle walking $(3.00$ $\pm 0.41 \mathrm{~J} \mathrm{~kg} / \mathrm{m})$. There were significant post hoc effects at $0.67 \mathrm{~m} / \mathrm{s}(\mathrm{p}<0.004 ; \mathrm{n}=8)$ and $0.84 \mathrm{~m} / \mathrm{s} \quad(\mathrm{p}<0.001 ; \mathrm{n}=8)$, where the energy cost of treadmill walking was higher than that of shuttle walking. Again, this pattern was reversed at higher walking speeds of $1.52 \mathrm{~m} / \mathrm{s} \quad(\mathrm{p}<0.042)$ and $1.69 \mathrm{~m} / \mathrm{s} \quad(\mathrm{p}<0.007)$ where shuttle walking had a greater energy cost per metre (for the $n=7$ patients achieving this level) than treadmill walking.

\section{DISCUSSION}

This is the first comparative investigation of the metabolic demands and energy cost per metre walking of incremental treadmill walking and shuttle walking in cardiac rehabilitation patients. We found differences in the oxygen requirements and energy cost of shuttle and treadmill walking large enough to suggest results from these exercise modalities should not be pooled in any future analyses.

Economy and energy requirements recorded during level 1 are difficult to interpret as they are most affected by oxygen kinetics and patients' unusually long stance phase during their gait cycle at this very slow walking speed and were excluded from our figures. The change in walking energy cost per metre on the treadmill show the expected pattern. Slow speeds are associated with higher cost per metre, which decreases as optimal (comfortable) walking speed approaches. Continuing to increase walking speed above this pace requires a greater cost per metre. In contrast to this, the energy cost per metre in shuttle walking decreases only very little and only following the first (very slow) walking pace in the initial stage. The energy cost then increases stage-by-stage throughout the protocol. The cost is only consistent between treadmill and shuttle walking between 1.2 and $1.4 \mathrm{~m} / \mathrm{s}$ (close to comfortable walking speed) and the increase in energy requirements is much greater in shuttle walking. Based on these pilot data, we intend to report cardiorespiratory fitness values separately according to test modality and recommend this practice to others.

The classical description of the energy cost during locomotion is of a U-shaped relationship ${ }^{18}$-as speed increases or decreases from the optimal $\left(1.11-1.3 \mathrm{~m} / \mathrm{s}^{18-20}\right)$ the energy cost of locomotion increases. For the treadmill protocol our data support this relationship. At slow speeds $(0.6-0.8 \mathrm{~m} / \mathrm{s})$, energy cost was greater than at optimal speeds $(1.2-1.4 \mathrm{~m} / \mathrm{s})$. As walking speed increased $(1.6-1.8 \mathrm{~m} / \mathrm{s})$ the energy cost again began to increase. This is comparable to Berryman et al, ${ }^{21}$ who reported a similar energy cost pattern for their 
participants (healthy elderly aged $68.9 \pm 4.6$ years) when walking on a treadmill at speeds ranging from 0.67$1.56 \mathrm{~m} / \mathrm{s}$ and the optimal walking speed was $1.33 \mathrm{~m} / \mathrm{s}$. Furthermore, our results also suggest that at lower speeds $(0.50-0.84 \mathrm{~m} / \mathrm{s})$, the energy cost of walking on a treadmill is greater than on the ground. Berryman et $a l^{21}$ also showed that there was greater energy cost of treadmill walking compared with ground walking at all the speeds they tested. The reason for the increased energy cost may be due to a greater need for stabilisation via muscular contraction while treadmill walking than when walking on the ground. ${ }^{21}$

Conversely, the oxygen requirements of shuttle walking are comparatively higher from level $7(1.52 \mathrm{~m} /$ s) onwards than for treadmill walking at the same speed. The requirements are also much higher $(18 \mathrm{~mL} / \mathrm{kg} /$ min) than the value predicted by the American College of Sports Medicine (ACSM) walking speed equations ${ }^{6}$ $(12.6 \mathrm{~mL} / \mathrm{kg} / \mathrm{min})$ that are used to estimate cardiorespiratory fitness from ISWT performance. ${ }^{4}$ In addition to differences in oxygen requirements of ground and treadmill walking, shuttle walking may have a higher cost due to repeated acceleration/deceleration phases or the negotiation of turns. ${ }^{7}$ We propose, therefore, that any clinical cut-offs for walking tests should be developed using the same testing modality as those for which they are proposed for use in (ie, treadmill or ground).

Cardiac patients' exercise capacity is commonly expressed as METs. We calculated metabolic cost in METs (gross $\dot{\mathrm{V}} \mathrm{O}_{2}[\mathrm{~mL} / \mathrm{kg} / \mathrm{min}] / 3.5$ ) and compared MET values at all ISWT stages with those reported previously $^{4}$ and the ACSM-predicted values (table 1). It should be noted that the values predicted using the ACSM walking equations by Woolf-May and Ferret ${ }^{4}$ are incorrect. The MET values they reported in cardiac patients are almost double the predicted values using the ACSM equations and much higher than those reported presently. Woolf-May and Ferrett's ${ }^{4}$ MET values further appear anomalous as they are more than double those of age-matched controls and significantly higher than recently reported values in cardiac patients during the ISWT. ${ }^{7}$ These latter values ${ }^{7}$ do, however, broadly agree with those presently reported.

Current recommendations suggest patients be classed as high risk if their exercise capacity is $<5$ METs. Failure to reach this criterion standard may lead to patients being prevented from entering community-based rehabilitation. ${ }^{22}$ Woolf-May and Ferret's ${ }^{4}$ suggestion that ISWT level 4 elicits a 5 MET energy cost in cardiac patients is inconsistent with recent data from Woolf-May and Meadows ${ }^{7}$ and those of the present study, both of which suggest the 5 MET threshold is nearer level 7 or 8 .

Fitter patients can be successfully 'fast tracked' to community rehabilitation, saving capacity and money to the health providers. ${ }^{23}$ However, where exactly in the ISWT protocol this threshold occurs should be determined in a larger, more representative cohort of cardiac patients.

Beyond level $7(1.52 \mathrm{~m} / \mathrm{s}, 3.8 \mathrm{mph})$ shuttle walking incurred an additional extra energy cost compared with treadmill walking, which may make it difficult to show small improvements in functional capacity if reported as estimated MET values. The exercise capacity of cardiac patients measured before outpatient rehabilitation tends to be lower when estimated from ISWT $^{24}$ than when standard treadmill protocols are used. ${ }^{25} 26$

\section{Study limitations and conclusions}

Along with sample size, this study is also limited due to including predominantly male patients and indeed only including data from male participants at the highest

Table 1 Comparison of predicted values, published values and measured metabolic cost (METs) of the incremental shuttle-walking test

\begin{tabular}{|c|c|c|c|c|c|}
\hline $\begin{array}{l}\text { ISWT protocol } \\
\text { level }\end{array}$ & $\begin{array}{l}\text { Walking } \\
\text { speed }(\mathrm{m} / \mathrm{s})\end{array}$ & $\begin{array}{l}\text { ACSM predicted } \\
\text { METs }\end{array}$ & $\begin{array}{l}\text { Published } \\
\text { ISWT METs }\end{array}$ & $\begin{array}{l}\text { Recorded METs: } \\
\text { treadmill walking } \\
\text { Mean (range) }\end{array}$ & $\begin{array}{l}\text { Recorded METs: } \\
\text { shuttle walking } \\
\text { Mean (range) }\end{array}$ \\
\hline 1 & 0.50 & 1.9 & 3.0 & $2.3(1.6-2.6)$ & $2.0(1.6-2.2)$ \\
\hline 2 & 0.67 & 2.1 & 3.7 & $3.3(2.8-4.0)$ & $2.7(2.5-3.1)$ \\
\hline 3 & 0.84 & 2.4 & 4.4 & $3.6(3.1-4.3)$ & $3.1(2.8-3.3)$ \\
\hline 4 & 1.01 & 2.7 & 5.1 & $3.8(3.2-4.6)$ & $3.6(3.2-3.8)$ \\
\hline 5 & 1.18 & 3.0 & 5.9 & $4.0(3.6-4.7)$ & $4.0(3.6-4.6)$ \\
\hline 6 & 1.35 & 3.3 & 6.6 & $4.4(4.3-5.9)$ & $4.4(4.0-4.9)$ \\
\hline 7 & 1.52 & 3.6 & 7.3 & $5.0(4.6-6.2)$ & $5.3(4.8-5.6)$ \\
\hline 8 & 1.69 & 3.9 & 8.0 & $5.5(5.0-6.7)^{*}$ & $6.1(5.7-6.6)^{*}$ \\
\hline 9 & 1.86 & 4.2 & 8.7 & - & - \\
\hline 10 & 2.03 & $4.5 / 7.9^{\star \star}$ & 9.4 & - & - \\
\hline 11 & 2.20 & $4.8 / 8.5^{\star \star}$ & 10.2 & - & - \\
\hline 12 & 2.37 & $5.1 / 9.1^{\star *}$ & 10.9 & - & - \\
\hline
\end{tabular}


walking speeds. The comparison of treadmill and shuttle walking may have been improved by increasing treadmill gradient, as is common practice. We omitted to do this for comparability with previous work. ${ }^{46}$ The accuracy of energy costs calculations would also be improved by including a resting metabolic measure pre-exercise instead of an assumed value of $4 \mathrm{~mL} / \mathrm{kg} / \mathrm{m} .^{9}$

In conclusion, the ISWT may have clinical utility as a measure of functional capacity to use in exercise prescription and patient monitoring, but we question its use as an estimate of cardiorespiratory fitness in cardiac patients. Importantly, the ACSM walking equations grossly underestimate the actual energy cost of shuttle walking and should not be used in research or clinical practice. Our comparison using METs also reveals that some published ${ }^{4}$ estimates of the ISWT's energy cost in cardiac patients appear erroneously high. Given these two shortcomings, we strongly warn against clinical decision-making or patient risk stratification based on achieving the 5 MET threshold estimated using the ISWT. We recommend a more accurate assessment of the ISWT's energy cost be performed in a larger, more generalisable sample of cardiac patients.

Contributors GRHS, RB and MJDT devised the experimental design. MA and FC collected and analysed the data. RB performed the metabolic modelling and advanced statistical analysis. GRHS and MJDT drafted the manuscript. $\mathrm{RB}, \mathrm{MA}$ and FC revised the manuscript. All authors contributed to the final preparation and drafting of the manuscript.

Funding This research received no specific grant from any funding agency in the public, commercial or not-for-profit sectors.

Competing interests None.

Patient consent Obtained.

Ethics approval University of Essex.

Provenance and peer review Not commissioned; externally peer reviewed.

Data sharing statement No additional data are available.

Open Access This is an Open Access article distributed in accordance with the Creative Commons Attribution Non Commercial (CC BY-NC 4.0) license, which permits others to distribute, remix, adapt, build upon this work noncommercially, and license their derivative works on different terms, provided the original work is properly cited and the use is non-commercial. See: http:// creativecommons.org/licenses/by-nc/4.0/

\section{REFERENCES}

1. Pepera G, McAllister J, Sandercock G. Long-term reliability of the incremental shuttle walking test in clinically stable cardiovascular disease patients. Physiotherapy 2010;96:222-7.
2. Woolf-May K, Bird S. Physical activity levels during phase IV cardiac rehabilitation in a group of male myocardial infarction patients. $\mathrm{Br} \mathrm{J}$ Sports Med 2005;39:e12; discussion e12.

3. Fowler SJ, Singh S. Reproducibility and validity of the incremental shuttle walking test in patients following coronary artery bypass surgery. Physiotherapy 2005;91:22-7.

4. Woolf-May K, Ferrett D. Metabolic equivalents during the 10-m shuttle walking test for post-myocardial infarction patients. $\mathrm{Br} J$ Sports Med 2008;42:36-41; discussion 41.

5. Sandercock GR, Cardoso F, Almodhy M, et al. Cardiorespiratory fitness changes in patients receiving comprehensive outpatient cardiac rehabilitation in the UK: a multicentre study. Heart 2013;99:785-90.

6. ACSM. ACSM's guidelines for exercise testing and prescription. 8th edn. Philadelphia: Lippincott Williams \& Wilkins, 2010.

7. Woolf-May K, Meadows S. Exploring adaptations to the modified shuttle walking test. BMJ Open 2013;3:pii:e002821.

8. SIGN. SIGN 57 cardiac rehabilitation. A national clinical guideline. Edinburgh: Royal College of Physicians, 2002.

9. Lentner C, ed. Giegy scientific tables. 7th edn. Basel: Ciba-Geigy, 1985:225-8.

10. Beneke R, Meyer K. Walking performance and economy in chronic heart failure patients pre and post exercise training. Eur J Appl Physiol Occup Physiol 1997;75:246-51.

11. di Prampero PE. The energy cost of human locomotion on land and in water. Int J Sports Med 1986;7:55-72.

12. Bobbert AC. Energy expenditure in level and grade walking. J Appl Physiol 1960;15:1015-21.

13. Ralston HJ. Energy-speed relation and optimal speed during level walking. Int $Z$ Angew Physiol 1958;17:277-83.

14. van der Walt $\mathrm{WH}$, Wyndham $\mathrm{CH}$. An equation for prediction of energy expenditure of walking and running. J Appl Physiol 1973;34:559-63.

15. Zaciorskij VM. Biomechanische Grundlagen der Ausdauer. Berlin: Sportverlag Berlin, 1987.

16. Zarrugh MY, Todd FN, Ralston HJ. Optimization of energy expenditure during level walking. Eur J Appl Physiol Occup Physiol 1974;33:293-306.

17. Brueckner JC, Atchou G, Capelli C, et al. The energy cost of running increases with the distance covered. Eur J Appl Physiol Occup Physiol 1991;62:385-9.

18. Workman JM, Armstrong BW. Metabolic cost of walking: equation and model. J Appl Physiol 1986;61:1369-74.

19. Bunc V, Dlouha R. Energy cost of treadmill walking. J Sports Med Phys Fitness 1997;37:103-9.

20. Mian OS, Thom JM, Ardigo LP, et al. Metabolic cost, mechanical work, and efficiency during walking in young and older men. Acta Physiol (Oxf) 2006;186:127-39.

21. Berryman N, Gayda M, Nigam A, et al. Comparison of the metabolic energy cost of overground and treadmill walking in older adults. Eur J Appl Physiol 2012;112:1613-20.

22. BACR. Standards and Core Components for Cardiac Rehabilitation (2007). Secondary Standards and Core Components for Cardiac Rehabilitation (2007). 2007. http://www.bcs.com/documents/affiliates/ bacr/BACR\%20Standards\%202007.pdf

23. Robinson HJ, Samani NJ, Singh SJ. Can low risk cardiac patients be 'fast tracked' to phase IV community exercise schemes for cardiac rehabilitation? A randomised controlled trial. Int J Cardiol 2011;146:159-63.

24. Almodhy MY, Sandercock GR, Richards L. Changes in cardiorespiratory fitness in patients receiving supervised outpatient cardiac rehabilitation either once or twice a week. Int J Cardiol 2012;160:215-16

25. Sharma A, McLeod AA. Cardiac rehabilitation after coronary artery bypass graft surgery: its effect on ishcaemia, functional capacity and a mulitvariate index of prognosis. Coron Health Care 2001;5:189-93.

26. Sandercock G, Hurtado V, Cardoso F. Changes in cardiorespiratory fitness in cardiac rehabilitation patients: a meta-analysis. Int $J$ Cardiol 2013;167:894-902. 